\title{
Electrokinetic remediation of soil polluted with insoluble organics using biological permeable reactive barriers: Effect of periodic polarity reversal and voltage gradient
}

Esperanza Mena ${ }^{1}$, José Villaseñor ${ }^{1, *}$, Manuel A. Rodrigo ${ }^{2}$ and Pablo Cañizares ${ }^{2}$

(1) Chemical Engineering Department. Research Institute for Chemical and Environmental Technology (ITQUIMA). University of Castilla La Mancha, 13071, Ciudad Real, Spain.

(2) Chemical Engineering Department. Faculty of Chemical Sciences and Technology. University of Castilla La Mancha, 13071, Ciudad Real, Spain.

*Corresponding author. Tel.: +34 9262953 00. E-mail: jose.villasenor@uclm.es

\begin{abstract}
This study investigated the remediation of clay soil polluted with low-solubility organics (diesel hydrocarbons) using a combined electrochemical-biological technology. Electrokinetic soil flushing was used, coupled with a biological permeable reactive barrier, which was placed into soil away from electrodes to prevent the negative effects of the acidic and basic fronts on the viability of microorganisms. Three two-week long batch experiments were performed in a lab-scale installation specifically designed to evaluate the remediation of polluted soil. The primary variable under study was the electric field applied to the soil $\left(0.5,1.0\right.$ and $\left.1.5 \mathrm{~V} \cdot \mathrm{cm}^{-1}\right)$, and the experimental procedure included daily polarity reversal of the electrodes and the addition of anionic surfactant in the electrode wells. The polarity reversal was observed to be a key strategy, which allowed adequate experimental conditions to be maintained in the soil (especially
\end{abstract}


temperature and $\mathrm{pH}$ ) for the success of the organic biodegradation process. The surfactant was evenly distributed across the soil, which helped the pollutant be transported. The biological activity was not limited to the barrier area but extended to the entire soil portion due to the microbial transport and growth far from the central barrier position. The voltage gradient did not have a strong influence on the measured experimental conditions (soil temperature, $\mathrm{pH}$ and moisture) but affected the electroosmotic flow. A higher diesel removal efficiency $(36 \%)$ was observed when using the higher voltage gradient $(1.5$ $\mathrm{V} \cdot \mathrm{cm}^{-1}$ ) after two weeks, which demonstrates a promising performance of the studied technology for a future real in-situ application.

\section{Keywords}

Non-soluble pollutants; Soil remediation; Electrokinetics; Biological permeable reactive barrier. 


\section{Introduction}

Soil pollution is a common problem related to accidental leaks during the transport, management or storage of hydrocarbons in underground deposits, distribution pipes, or oil refining processes. It is an important environmental issue because of the negative impact caused on soil and underground water due to the hazardous characteristics of the chemical constituents of fuel [1].

Currently, different technological options for fuel-polluted soil remediation are available, and they are based on thermal, chemical, physicochemical or biological fundamentals [2]. All of these technologies can be classified into two primary groups: in-situ and ex-situ treatments. In-situ technologies try to remediate the polluted soil without removing it from its original site, which is clearly a cheapest option. However, the mass and energy transport phenomena required to clean the soil are clearly disadvantaged. This limitation is especially important in low permeable soil.

Electrokinetic (EK) soil remediation is a remarkable technology that is especially recommended for the in-situ treatment of low permeability soils with low hydraulic conductivity values (i.e., polluted sites where hydrodynamic transport would not be suitable). EK remediation consists of the application of a direct electric current across electrodes placed in the polluted soil. This electric field cause different transport mechanisms [3], such as electromigration (movement of ions to the opposite electrodes), electrophoresis (movement of charged particles, including microorganisms, to the opposite electrodes), and electro-osmosis (movement of water to the cathode caused by superficially charged phenomena). Additionally, different electrochemical reactions occur, such as electrolysis and electrodeposition, so that the remediation process is the result of a complex set of mass transport phenomena and electrochemical reactions [4]. 
Previous works reported that EK remediation has been successful and cost-effective for removing different types of contaminants from low-permeability soils $[5,6]$.

To improve the remediation efficiency of the in-situ EK technology, there has been recent increased interest in combining EK methods with other conventional soil remediation technologies [7]. Particularly, one option is the combination of permeable reactive barriers (PRB) and EK treatments [8]. Generally, PRBs are the artificial media of a reactive material that is located in the soil in the direction of groundwater flow, so that the PRB intercepts the pollution plume and retains or eliminates the pollutants [9]. The subsurface pollution plume can be forced to flow through the PRB using natural hydraulic gradients or pumps. However, if the soil to be treated is extremely low permeable, the pollutants can be moved through the PRB by the EK mechanisms previously described. Different materials can be used to build PRBs. They are based on different underlying mechanisms (ion exchange with resin-type materials, chemical reduction using zerovalent metals, adsorption with granular activated carbon, etc.). Particularly, PRBs based on biological degradation are known as biological permeable reactive barriers (Bio-PRB) or biobarriers. A biobarrier can be a fixed bed culture bioreactor (i.e., a porous supporting material with a microbial biofilm attached on its surface) or a wet mixture of soil and activated sludge $[10,11,12]$.

Although the number of publications on this topic is increasing [7], the combination of EK methods and PRB treatments is relatively recent. Generally, the combination of EK methods and any biological technique (bioremediation) was previously known as “electrobioremediation" [13].

Furthermore, the EK remediation technology involves certain additional phenomena in the soil, such as electrical heating because of ohmic drops related to the low ionic conductivity of soil, removal of nutrients from the soil due to the EK transport processes, 
and strong $\mathrm{pH}$ gradients near the electrodes [14]. These phenomena can be a considerable inconvenience for the correct performance of the biological processes in the soil. The polarity reversal strategy (i.e., the periodic change in the polarity of the electric field applied to the soil) has been proposed as a simple solution for regulating changes in the $\mathrm{pH}$ and improving the distribution of ionic inorganic nutrients [15].

The goal of the current study is to investigate the effect of the voltage gradient on the remediation of a diesel hydrocarbon-polluted clay soil through a combined electrochemical-biological technology, i.e., using the EK system and a Bio-PRB included in the soil. Furthermore, the polarity reversal strategy has been included, and the electric field is the primary input to be characterized (voltage gradient). This study has been performed using lab-scale batch experiments, and it is supported by previous research performed by the same authors $[16,17]$.

\section{Materials and methods}

The materials, experimental procedures, and analytical methods have been described elsewhere in previous studies performed by the same authors $[16,17,18]$ and related to the same research programme. The following subsections only describe the important details.

\subsection{Electrokinetic installation}

The lab-scale set-up is shown in Figure 1 and it was used to simulate in the laboratory an in-situ real process. It was made of transparent methacrylate and divided into five compartments, and the central compartment contained the polluted soil and a $5 \mathrm{~cm}$ thickness biobarrier in the central position, separated by nylon mesh ( $0.5 \mathrm{~mm}$ mesh size). The electrode compartments were on one side of each section of polluted soil. They were also separated from the soil by a $0.5 \mathrm{~mm}$ nylon mesh. Each electrode compartment had an overflow to collect the water transported because the electroosmosis process. Graphite 
electrodes were located into the electrodic compartments and connected to the power supply (HQ Power, Gavere, Belgium) so that one of them constituted the anode and the other one the cathode. Dimensions of these electrodes, provided by Carbosystem (Madrid, Spain), were $10.0 \times 10.0 \times 1.0 \mathrm{~cm}^{3}$. In this way, they had the same cross section as the fraction of soil to be treated, which maintained a homogeneous distribution of current lines throughout the soil [16].

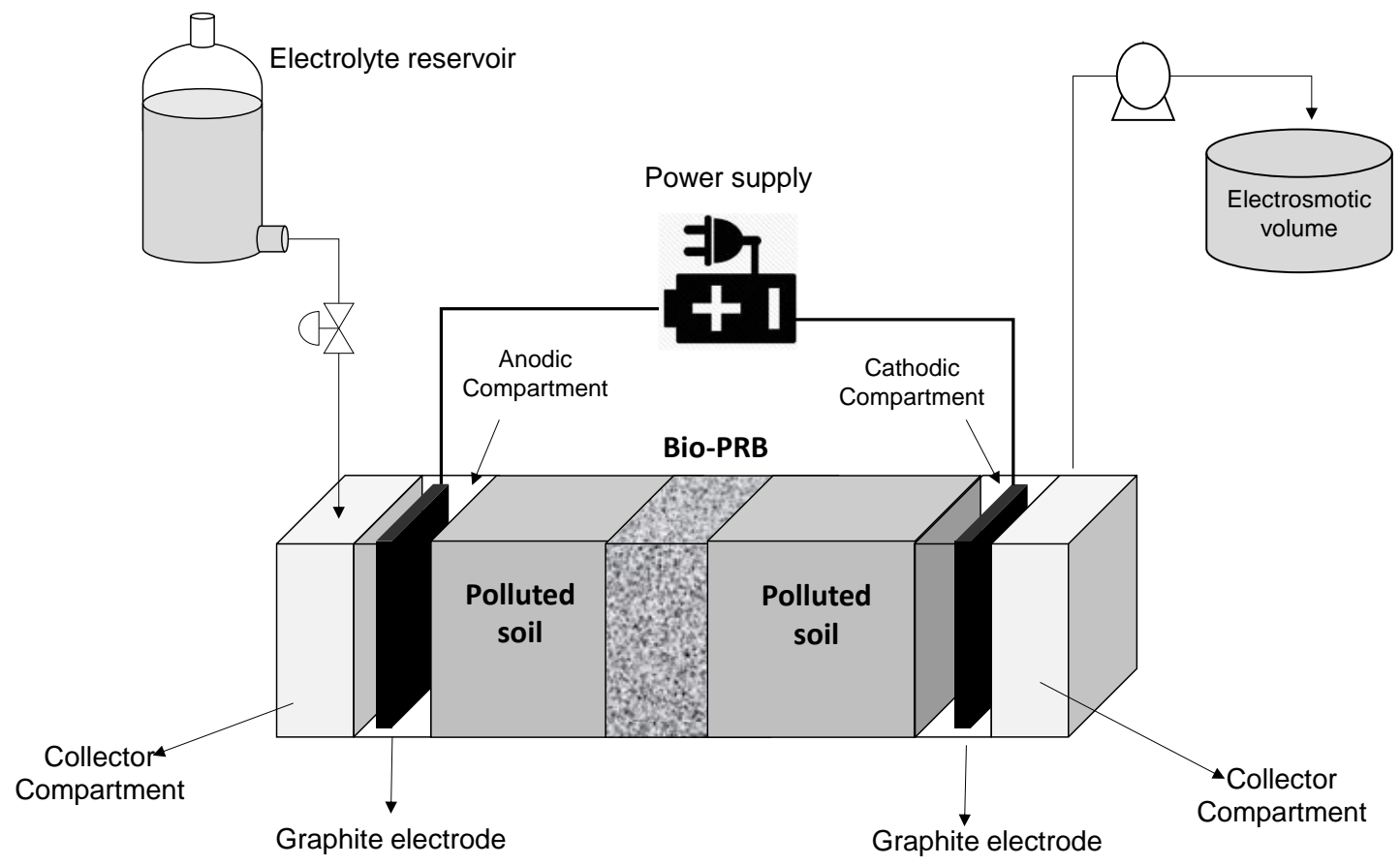

Fig.1. Experimental installation

\subsection{Materials}

Kaolinite was selected as a model for clay soil. The properties of this synthetic clay soil have been detailed in Table 1. The diesel fuel, which was used as the pollutant, was a conventional petroleum-derived fuel that was purchased from a petrol station. The fuel composition was obtained according to ASTM standard tests (ASTM D86, 2004), and the analysis showed that it was composed of $75 \%$ saturated hydrocarbons (primarily paraffins, including $\mathrm{n}-$, iso-, and cycloparaffins) and $25 \%$ aromatic hydrocarbons 
(including naphthalenes and alkylbenzenes). Additional details have been previously reported [18]. The soil was artificially polluted by diluting diesel in acetone before carefully distributing the solution in the corresponding amount of kaolinite. The solvent (acetone) and the higher volatile diesel fractions (approximately $6 \%$ of the initial amount of added diesel) were allowed to evaporate at room temperature for at least two days. The diesel concentration in the polluted soil at the beginning of the experiments was $10 \mathrm{~g} \cdot \mathrm{kg}^{-}$ 1 .

An inorganic salt solution was used as an anolyte/catholyte to moisten the soil and ensure electrical conductivity. It was composed of the following proportions: $30.36 \mathrm{mg} \cdot \mathrm{l}^{-1}$ of $\mathrm{NaNO}_{3}, 70 \mathrm{mg} \cdot \mathrm{l}^{-1}$ of $\mathrm{NaHCO}_{3}$ and $88.75 \mathrm{mg} \cdot \mathrm{l}^{-1}$ of $\mathrm{Na}_{2} \mathrm{SO}_{4}$. Additionally, an anionic surfactant (Sodium Dodecyl Sulphate, SDS, whose CMC is $8.2 \mathrm{mM}$ ) was used as a flushing agent in both electrode compartments. Considering that diesel is a non-polar pollutant and because the biological active area was placed in a central section of the installation, it was necessary for the surfactant to promote the transport of the diesel pollutant through the biobarrier [19].

The biobarrier consists of mixing of non-polluted kaolinite with the corresponding volume of raw biological activated sludge to obtain a similar moisture level as in the polluted soil. The sludge was obtained from the aerobic bioreactor of a domestic wastewater treatment plant. The sludge total volatile solids concentration was approximately $10 \mathrm{~g} \cdot \mathrm{L}^{-1}$ and the total volatile solids concentration in the Bio-PRB at the beginning of the treatment was $4.5 \mathrm{~g} \cdot \mathrm{kgSoil}^{-1}$. The activated sludge was previously supplemented with a Bushnell-Hass Broth (BHB) nutrient medium to ensure availability of inorganic nutrients for the biodegradation process. More details have been reported elsewhere [16].

\subsection{Experimental procedure}


The soil was compacted into the central compartment on both sides of the biobarrier. Then, the biobarrier was loaded into the central position (Figure 1). The clay soil was soaked with the electrolyte solution for a moisture content of $40 \%$. The inorganic salts solution, including a $2.38 \mathrm{~g} \cdot \mathrm{l}^{-1}$ SDS amount, was loaded into the cathodic/anodic compartment at the beginning of the experiments. Three batches of experiments with a two-week duration each were performed using potentiostatic conditions (i.e., setting a voltage gradient value that remained constant throughout the experiment), and the polarity reversal was applied every $24 \mathrm{~h}$. The variable under study was the electric field (voltage gradient), which was set at $0.5,1.0$ and $1.5 \mathrm{~V} \cdot \mathrm{cm}^{-1}$. Based on the length of the treated section $(20 \mathrm{~cm})$, the total value of the fixed voltage was $10.0,20.0$ and $30.0 \mathrm{~V}$.

\subsection{Sampling and analyses}

Soil temperature, electrical current density through the soil, and electro-osmotic water flow were monitored daily. Additionally, $\mathrm{pH}$, moisture content, and concentrations of microorganisms, diesel and SDS were measured in the soil at the beginning $(\mathrm{t}=0)$ and end $(t=14 \mathrm{~d})$ of the experiments. At the end of the experiments, soil samples were obtained from four longitudinal positions corresponding to four intermediate points between the anode and the cathode compartments. Hence, each intermediate longitudinal position also contained four sampling points distributed at different axial positions. The results indicate the average concentration of the four different samples obtained at the same longitudinal position, i.e., at the same distance from the electrodes.

The diesel hydrocarbon concentration was expressed as the Total Petroleum Hydrocarbon (TPH), and the concentration of microorganisms was expressed in Colony Forming Units (CFU) as reported by Mena Ramírez et al. [17]. Moisture and pH were measured as reported elsewhere [16]. The SDS concentration was measured using a previously reported specific photometric method [20]. 


\section{Results and discussion}

Figure 2 a depicts the variation in the current density (defined as the ratio between the current intensity and the cross section area of the soil columns) during the three soil remediation tests performed in this study. As expected, for a higher applied electric field, the resulting current density is higher. Slight oscillations in the value of the current density corresponding with the daily change in the polarity of the electric field applied were observed. These oscillations were more pronounced when applying higher values of electric field, which can be explained in terms of the change in the charge of the double layer, resulting in a high consumption of the capacitive part of the current associated to the discharging or recharging of the double layer [21], and the distribution around the soil particles of the ionic species present in the pore water. These ionic species are the contained in the composition of the solution used to moisten the soil and ensure its electrical conductivity, and the inorganic nutrients (BHB medium) added in the biobarrier to support the biological degradation process. However, it was not possible to determine what specific ions would be the main responsible of this phenomenon. By observing the full length of the tests, a slight decrease in the current density value was obtained, which may be explained in terms of the decrease in the conductivity of the soil compared to the initial conditions. It was observed (results not shown) that the soil conductivity clearly decreased during the experiments, and the decrease was greater at higher voltage values. 

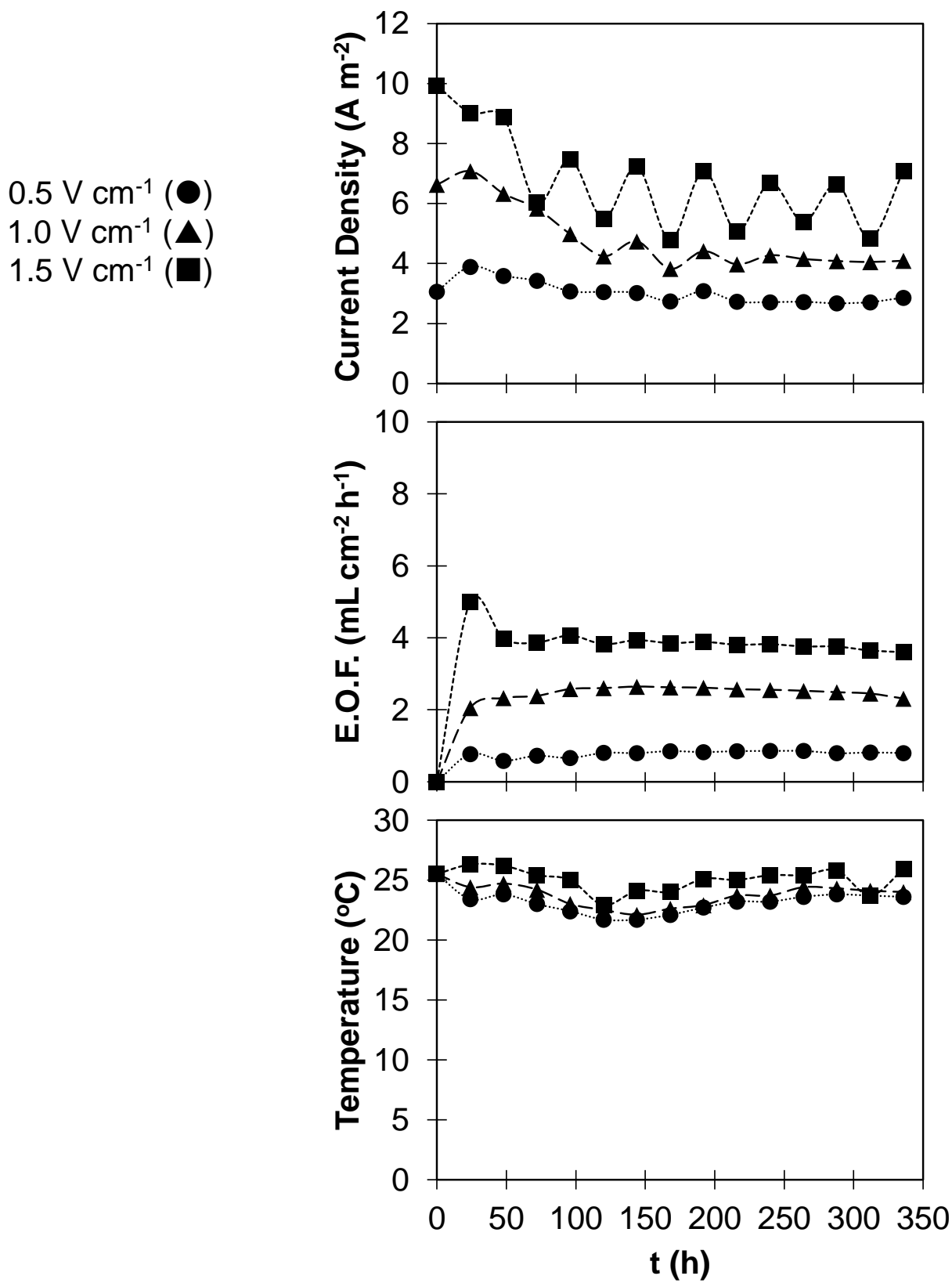

Fig. 2. Time-course of the (a) current density, (b) electro-osmotic flow, and (c) soil temperature during the experiments at $0.5 \mathrm{~V} \cdot \mathrm{cm}^{-1}(\mathbf{O}), 1.0 \mathrm{~V} \cdot \mathrm{cm}^{-1}(\mathbf{\Delta})$ and $1.5 \mathrm{~V} \cdot \mathrm{cm}^{-1}$ (ם).

Figure $2 \mathrm{~b}$ focuses on the variation in the electro-osmotic flow measured in the three experiments. According to the Helmholtz-Smoluchowski equation (Equation 1), the higher the electric field applied (Ex) the higher is the rate of the electro-osmotic flux 
(EOF), because the dielectric constant (D), vacuum permittivity $\left(\varepsilon_{0}\right)$, and fluid viscosity $(\mu)$ are expected to be almost the same in the four tests.

$$
E O F=\frac{-D \varepsilon_{0} Z}{\mu} E_{x}
$$

According to it, for a higher electric field, the resulting electro-osmotic flow was higher, even though linearity does not appear to be maintained. Thus, the slopes calculated for this equation are $1.57,2.48$ and 2.61 for the $0.5,1.0$ and $1.5 \mathrm{~V} \cdot \mathrm{cm}^{-1}$ electric fields, respectively. These values suggest that more complex processes are occurring in the soil, thus involving significant changes in the Z-potential $(\mathrm{Z})$ of the soil.

The polarity reversal strategy applied in this study causes reversal in the EOF direction, and it has been reported that it also results in a lower volume of water being transported to the electrode wells [22]. This is an important aspect from a biological perspective because it would improve the distribution of the nutrients and reduce the nutrients removed from the soil, thus allowing better performance of the pollutant's biodegradation process $[23,24]$.

Additionally, the changes in the soil temperature were monitored in these tests, as plotted in Figure 2c. In these experiments, due to the low values of the applied current densities, no large changes in temperature due to soil heating were detected. The temperatures were comparable in the three experiments, and only slightly higher values were detected in the experiment at the highest electric field. These temperature values are adequate for the performance of the biological process. Regarding the soil moisture levels, there were no changes during the experiments, and the initial value (40\%) was maintained in all cases. Thus, the results were not included in figure 3. To keep the moisture level is also very important for the proper functioning of the biological process.

Figure 3 presents the $\mathrm{pH}$ profile across the soil at the start and the end of the experiments. It can be observed that contrary to what is observed for other EK soil remediation 
strategies, the $\mathrm{pH}$ values remained constant after the treatment and close to neutrality, which is an important requirement for the success of the pollutant biodegradation process. No significant influence of the magnitude of the electric field was observed in the profiles. Therefore, it should be noted that different strategies have been reported in the literature to control the $\mathrm{pH}$ gradients, such as the polarity reversal or the buffer addition in the electrode wells [7, 25, 26, 27], and also the application of electric current only during short periods of time $\left(8 \mathrm{~h} \mathrm{~d}^{-1}\right)[28,29]$. However, the buffer addition approach could be an inconvenience because of the expected increase in the current density and excessive soil heating [5]. Thus, the application of the reversion of the electric field is not new, i.e., it is a well-known technique, and the results obtained in our study support their relevance and suitability to be combined with biological processes by the inconveniences in the conditions for the right performance of the microbial activity $[6,8,30]$. 

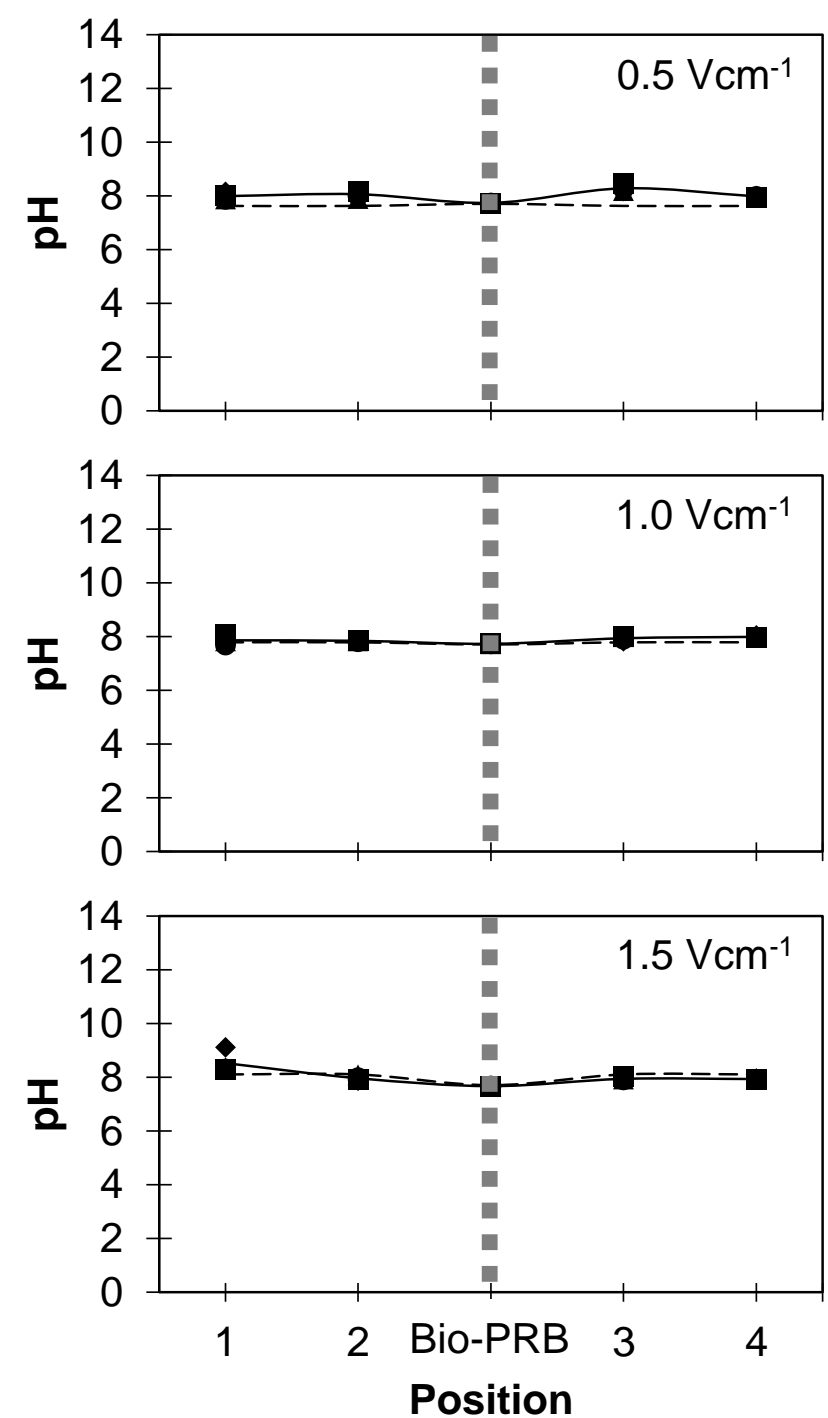

Fig. 3. pH profile in the soil at the start (- -$)$ and the end (-) of the experiments at $0.5,1.0$ and $1.5 \mathrm{~V} \cdot \mathrm{cm}^{-1}$. The line (-) is the average of the four values in the different axial positions (top right $(\bullet)$, top left $(\boldsymbol{\square})$, bottom right $(\mathbf{\Lambda})$, and bottom left $(\boldsymbol{O})$ ). 

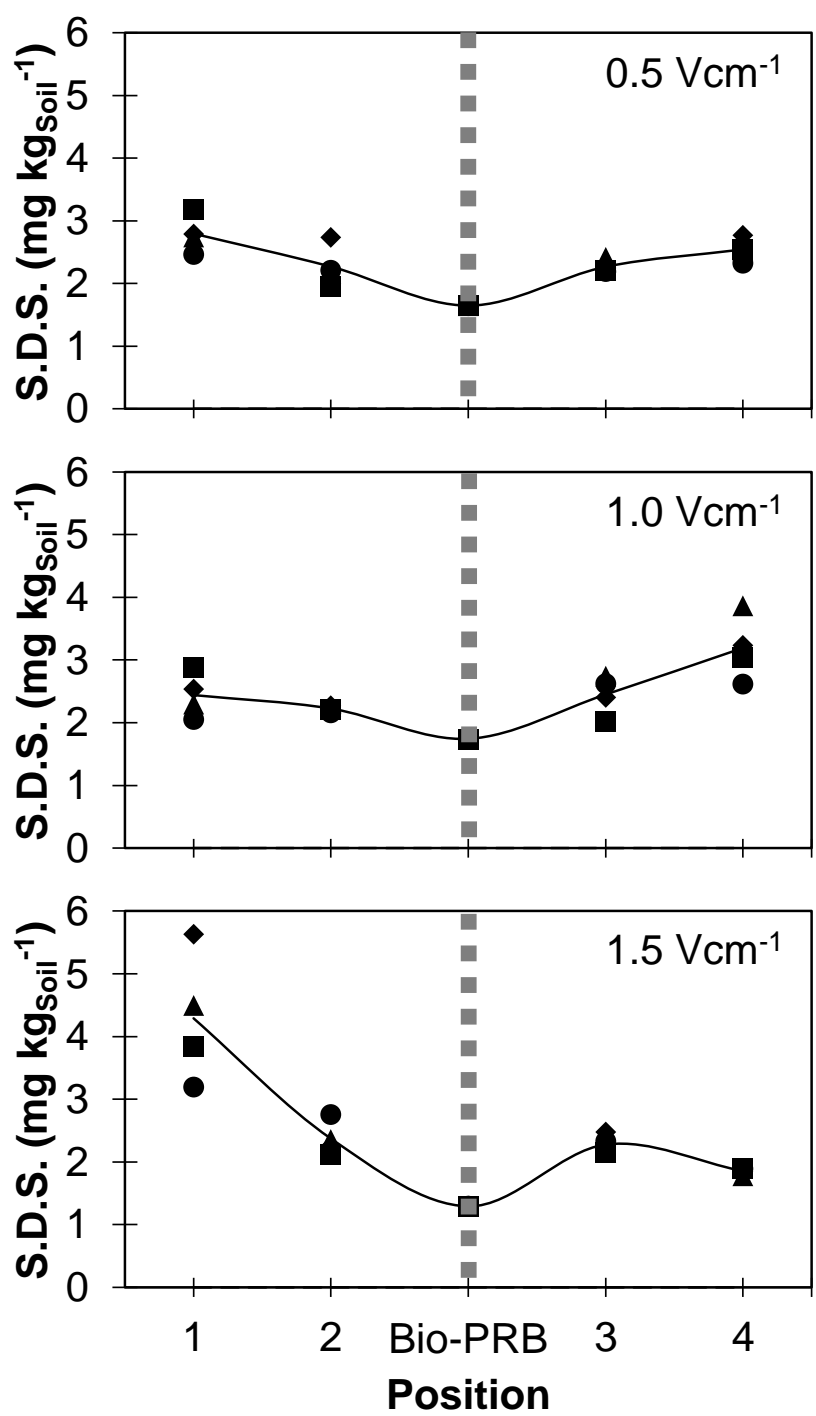

Fig. 4. Surfactant concentration profile in the soil at the end of the experiments at $0.5,1.0$ and $1.5 \mathrm{~V} \cdot \mathrm{cm}^{-1}$. The line is the average of the four values in the different axial positions (top right $(\bullet)$, top left $(\boldsymbol{\square})$, bottom right $(\mathbf{\Delta})$, and bottom left $(\boldsymbol{O})$ ).

Figure 4 illustrates the profiles of the surfactant concentration detected in the soil at the end of the experiments (there were no surfactant in the soil at the beginning). A similar profile was obtained in the three experiments, with slightly higher concentrations in the areas near the electrodic compartments, which is where the surfactant solution was added. These profiles were relatively symmetrical with respect to the central point, except for the third experiment $\left(1.5 \mathrm{~V} \cdot \mathrm{cm}^{-1}\right)$, in which the largest electric field was applied. In this 
case, higher concentrations of the surfactant were detected in the sections near the electrode working as an anode at the beginning of the experiment. Due to the anionic characteristics of the surfactant, it has been noted that the direction of migration of the micelles imposed at the beginning of the treatment could be predominant during the entire experiment. In the rest of experiments conducted at lower voltage values, the inertia in the electrokinetic transport was more easily modified as the experiment proceeded. However, an accurate surfactant distribution was obtained over the entire soil, which has contributed to the success of the treatment: the EK-PRB technology treating non-polar pollutants needs the flushing fluids-containing surfactant to be efficient because these species assist in the transport of such pollutants (i.e., diesel oil) to the barrier [19]. In the experiments demonstrated in the current study, the surfactant solution was evenly distributed over the entire soil, and it was expected that the surfactant would be transported in both directions due to the polarity reversal, which would cause the diesel flow to pass across the central Bio-PRB repeatedly.

Figure 5 presents the profiles of the microbial concentrations detected in the soil at the end of the experiments. The native soil microbial concentration at the start of the experiments was negligible. It should be noted that we are using a laboratory material (clay) as artificial soil. Moreover, the activated sludge was only introduced at the central Bio-PRB position at the beginning. A relatively homogeneous concentration profile was again observed across the soil, even near the electrodes. This result could be related to two phenomena: the microbial transport due to electrophoresis and the microbial growth far from the central position due to substrate (diesel) consumption. The lower population observed at the higher electric field in the areas near the electrodes could be related to the extreme change in the $\mathrm{pH}$ in the wells. 

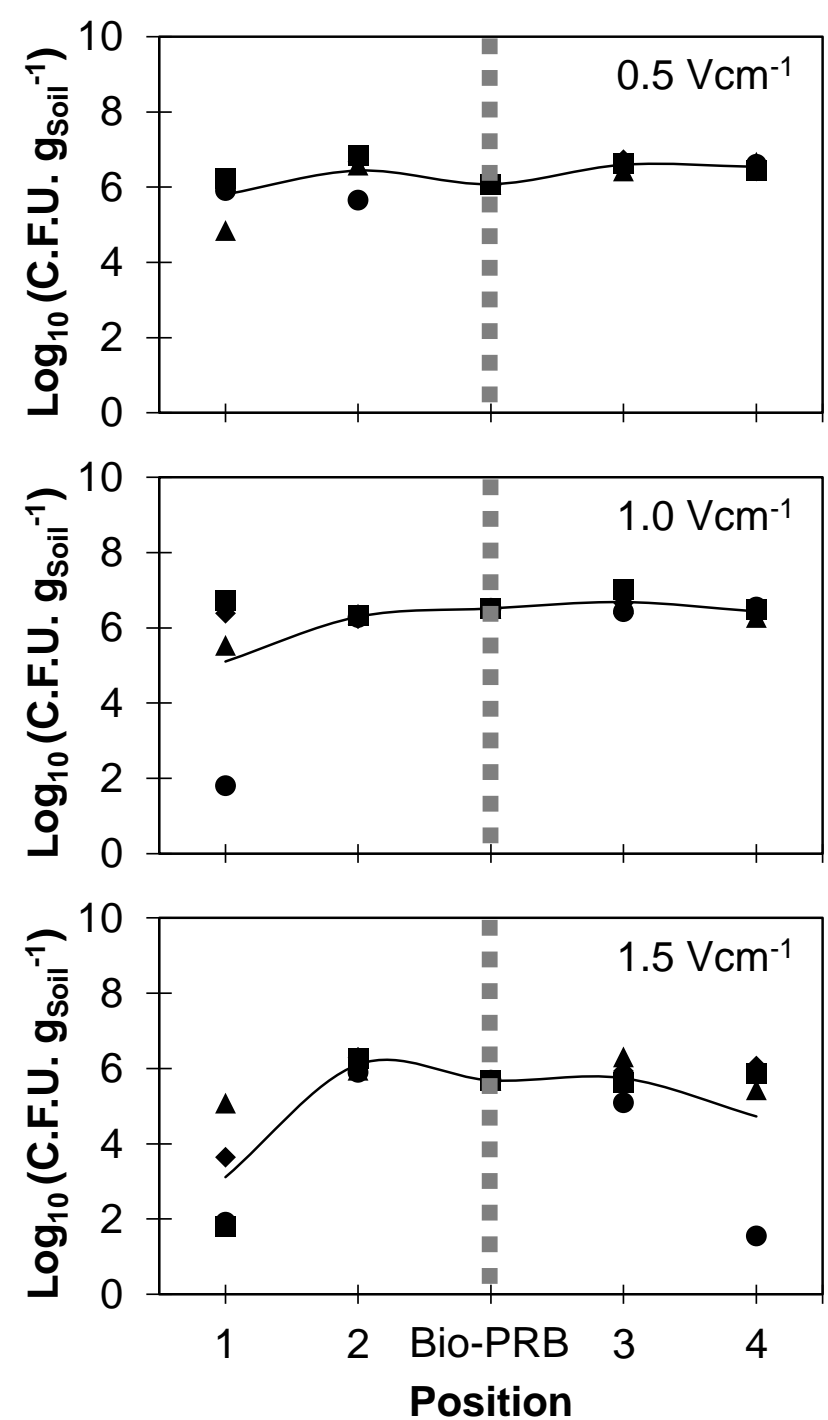

Fig. 5. Microbial concentration profile in the soil at the end of the experiments at $0.5,1.0$ and $1.5 \mathrm{~V} \cdot \mathrm{cm}^{-1}$. The line is the average of the four values in the different axial positions (top right $(\bullet)$, top left $(\boldsymbol{\square})$, bottom right $(\mathbf{\Delta})$, and bottom left $(\boldsymbol{O})$ ).

In EK remediation systems, the microbial transport has been reported to preferentially tend towards the cathode because of the surface charge of the microorganisms [31]. However, because of the polarity reversal strategy applied in the current study, the transport is expected in both directions from the biobarrier. Considering this enhanced transport of microorganisms, the microbial growth and activity are not expected to be 
limited to the biobarrier and can be possible further away. Because once the microorganisms reach zones far from the central position as the experimental conditions become suitable (adequate values of temperature, $\mathrm{pH}$, diesel and nutrients distribution), they can degrade the pollutants efficiently. Similarly, a biostimulation option could also be possible (i.e., growth of native microorganisms initially present in the soil), even though the authors consider it not to be relevant to this study because of the use of synthetic soil.

The extended activity zone of the microorganisms has been previously described. Thus, although the objective of the PRB is that the pollutant is forced to flow across an immobilized reactive PRB material where microorganisms are in the best conditions for attaining good performances, it has also been reported that the activity of the microorganism is not located only in the central Bio-PRB position because microorganisms are present in wide areas of the soil despite using non-fixed activated sludge [16] and microbial biofilm cultures fixed to a gravel bed [17]. The central BioPRB location was always the recommended position to be sufficiently far from the strong $\mathrm{pH}$ values near the electrodes; however, this precaution would not be necessary in the current study because of the polarity reversal option.

Figure 6 depicts the diesel concentration profiles at the start and the end of the experiments. The removal of diesel can be observed in all of the cases; however, the efficiencies are different based on the soil position and the voltage gradient used. 

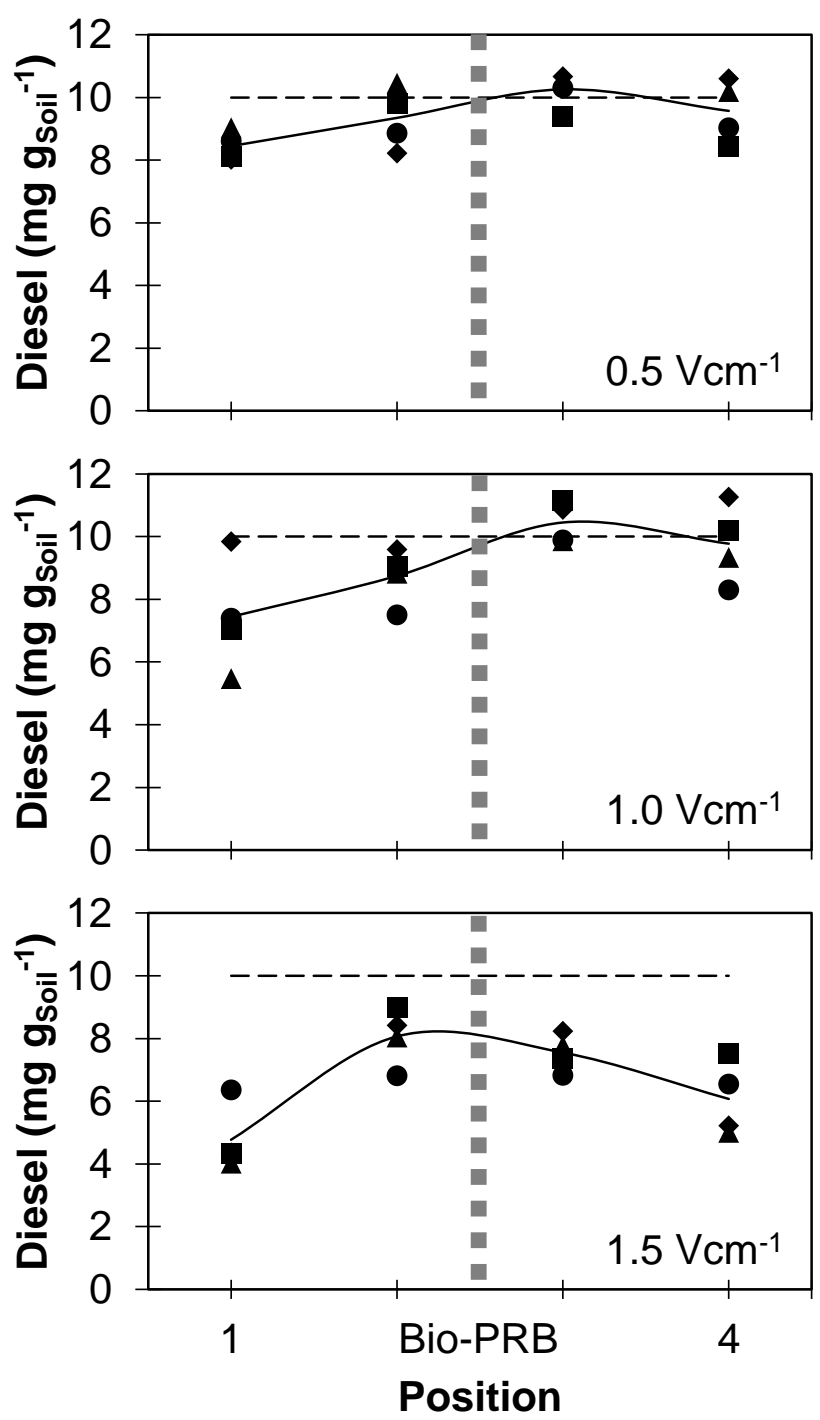

Fig. 6. Diesel concentration profile in the soil at the start (- ) and the end ( - ) of the experiments at $0.5,1.0$ and $1.5 \mathrm{~V} \cdot \mathrm{cm}^{-1}$. The line (-) is the average of the four values in the different axial positions (top right $(\bullet)$, top left $(\boldsymbol{\square})$, bottom right $(\mathbf{A})$, and bottom left $(\bigcirc))$.

First, it should be noted that the diesel removal from the soil has been related only to the microbial activity and not to any other mechanisms because the possible contribution of the hydrocarbon volatilization process has already been considered (subsection 2.2) and diesel was not removed by the EK transport as it was not detected in the electrode wells at the end of the experiments. Indeed, diesel was not detected in the wells, during almost 
the entire duration of the experiments, except at the beginning, when very low concentrations were detected. It was supposed that low amounts of diesel were transported to the wells at the beginning by EK movement, but the movement was slow and the flow direction was changing every $24 \mathrm{~h}$ because of the polarity reversal. These conditions allowed the presence of very low concentrations of diesel in the wells at the beginning, while there were no degradation in the areas near the electrodes. However, at the end of the experiments, the biodegradation process was active in the whole soil (as the microbial profiles demonstrated) and the combination of biodegradation and the mixing effect of the polarity reversal finally completely removed diesel from wells. The microbial distribution and growth across the soil would agree with these statements. However, there is a clear influence of the voltage gradient. A diesel removal efficiency less than 0.5 or $1.0 \mathrm{~V} \cdot \mathrm{cm}^{-1}$ is low and not homogeneous, and the removal was only obtained in one half of the soil portion (the left part in the figure, which corresponds to the initial anode position). This behaviour is difficult to justify, and the same effect previously stated has been assumed to explain the differences in the higher surfactant concentration near the anode. Conversely, a diesel removal efficiency less than $1.5 \mathrm{~V} \cdot \mathrm{cm}^{-}$ ${ }^{1}$ is higher, and it has been achieved in the entire soil.

The higher removal efficiency under $1.5 \mathrm{~V} \cdot \mathrm{cm}^{-1}$ was obtained despite there were no important differences in the SDS profiles (figure 4) or microbial profiles (figure 5) at the end of the experiments. Regarding the surfactant profiles, it must be noted that the objective is not to reach higher SDS concentrations with higher voltages, but is to obtain a correct and homogeneous distribution. It could be supposed that higher voltages would accelerate the surfactant movement and it would suggest a better mixing effect. Based on these results, it is believed that the higher electric field improved the EK transport processes because it caused a faster homogenization effect compared to the lower voltage 
experiments. Regarding the microbial profiles, large differences in microbial growth were not expected as the differences in the organic removal efficiencies were not large and because the biological process didn`t work under completely aerobic conditions (which would imply higher biomass yields). The best average diesel removal efficiency after two weeks was approximately $36 \%$, which is approximately $50 \%$ of the diesel biodegradable fraction, because it was necessary to take into account the inert (0.3) and the biodegradable (0.7) fractions that were determined in a previous work [17]. Compared to the previous works published by the authors $[16,17]$, the present work finally shows a better-optimized EK-BioPRB technology and it gives information about the influence of the voltage gradient.

\section{Conclusions}

It is possible to remove diesel hydrocarbons from a clay soil using an EK-BioPRB technology. According to the well-known effects of the polarity reversal strategy, it has been observed that polarity reversal allows maintaining adequate experimental conditions for the biological process, and the surfactant addition appears to be necessary to help the diesel transport into the soil. The voltage gradient does not have a strong influence on several experimental conditions (soil $\mathrm{pH}$, temperature and moisture); but it affects the EOF. It has been observed that a higher voltage gradient results in a higher removal efficiency. Compared to other PRB systems, the biological activity has been extended to the entire soil area because of the microbial transport and growth far from the central BioPRB position. The maximum removal efficiency obtained was $36 \%$ after two weeks.

\section{Acknowledgements}

The authors acknowledge funding support from the EU and Spanish Government through the MINECO Project CTM2013-45612-R and INNOCAMPUS. 


\section{References}

[1] M. Fingas, Oil spills and their cleanup: introduction, in: Encyclopedia of Environmental Management, Taylor and Francis, New York, 2013, Published online 21 Nov 2013, pp. 1-17.

[2] A.A. Juwarkar, S.K. Singh, A. Mudhoo. A comprehensive overview of elements in bioremediation, Rev. Environ. Sci. Biotechnol. 9 (2010) 215-288.

[3] J. Virkutyte, M. Sillanpaa, P. Latostenmaa, Electrokinetic soil remediation - critical overview, Sci. Total Environ. 289 (2002) 97-121.

[4] A.T. Yeung, Contaminant extractability by electrokinetics, Environ. Eng. Sci. 23 (2006) 202-224.

[5] K.R. Reddy, C. Cameselle, Overview of electrochemical remediation technologies, in: K.R. Reddy, C. Cameselle (Eds.), Electrochemical Remediation Technologies for Polluted Soils, Sediments and Groundwater, John Wiley \& Sons Inc., Hoboken, 2009, pp. 223-227.

[6] M.A. Rodrigo, N. Oturan, M.A. Oturan, Electrochemically Assisted Remediation of Pesticides in Soils and Water: A Review, Chem. Rev. 114 (2014) 8720-8745.

[7] A.T. Yeung, Y.Y. Gu, A review on techniques to enhance electrochemical remediation of contaminated soils, J. Hazard. Mater. 195 (2011) 11-29.

[8] C. Weng, Coupled electrokinetics-permeable reactive barriers, in: K.R. Reddy, C. Cameselle (Eds.), Electrochemical Remediation Technologies for Polluted Soils, Sediments and Groundwater, John Wiley \& Sons Inc., Hoboken, 2009, pp. 483-503.

[9] E.K. Nyer, In situ treatment technology, Lewis Publishers, Boca Raton, Florida, 2001. [10] C.M. Kao, S.C. Chen, J.K. Liu, Development of a biobarrier for the remediation of PCE-contaminated aquifer, Chemosphere 43 (2001) 1071-1078. 
[11] C.M. Kao, S.C. Chen, J.Y. Wang, Y.L. Chen, S.Z. Lee, Remediation of PCEcontaminated aquifer by an in situ two-layer biobarrier: Laboratory batch and column studies, Water Res. 37 (2003) 27-38.

[12] S. Saponaro, M. Negri, E. Sezenna, L. Bonomo, C. Sorlini, Groundwater remediation by an in situ biobarrier: A bench scale feasibility test for methyl tert-butyl ether and other gasoline compounds, J. Hazard. Mater. 167 (2009) 545-552.

[13] L.Y. Wick, L. Shi, H. Harms, Electro-bioremediation of hydrophobic organic soilcontaminants: A review of fundamental interactions, Electrochim. Acta 52 (2007) 34413448.

[14] Y.B. Acar, R.J. Gale, A.N. Alshawabkeh, R.E. Marks, S. Puppala, M. Bricka, R. Parker, Electrokinetic remediation: Basics and technology status, J. Hazard. Mater. 40 (1995) 117-137.

[15] D. Huang, S. Guo, T. Li, B. Wu, Coupling interactions between electrokinetics and bioremediation for pyrene removal from soil under polarity reversal conditions. Clean: Soil, Air, Water 41 (2013) 383-389.

[16] E. Mena, C. Ruiz, J. Villaseñor, M.A. Rodrigo, P. Cañizares, Biological permeable reactive barriers coupled with electrokinetic soil flushing for the treatment of diesel polluted clay soil, J. Hazard. Mater. 283 (2015), 131-139.

[17] E. Mena Ramírez, C. Sáez Jiménez, J. Villaseñor Camacho, M.A. Rodrigo Rodrigo, P. Cañizares Cañizares, Feasibility of coupling permeable biobarriers and electrokinetics for the treatment of diesel hydrocarbons polluted soils, Electrochim. Acta 181 (2015) 192199.

[18] E. Moliterni, R.G. Jiménez-Tusset, M. Villar Rayo, L. Rodriguez, F.J. Fernández, J. Villaseñor, Kinetics of biodegradation of diesel fuel by enriched microbial consortia from polluted soils, Int. J. Environ. Sci. Tech. 9 (2012) 749-758. 
[19] R. López-Vizcaíno, J. Alonso, P. Cañizares, M.J. León, V. Navarro, M.A. Rodrigo,

C. Sáez, Removal of phenanthrene from synthetic kaolin soils by electrokinetic soil flushing, Sep. Purification Technol. 132 (2014) 33-40.

[20] E. Jurado, M. Fernández-Serrano, J. Núñez-Olea, G. Luzón, M. Lechuga, Simplified spectrophotometric method using methylene blue for determining anionic surfactants: Applications to the study of primary biodegradation in aerobic screening tests, Chemosphere 65 (2006) 278-285.

[21] J. Röhrs, G. Ludwig, D. Rahner, Electrochemically induced reactions in soils - A new approach to the in situ remediation of contaminated soils? Part 2: Remediation experiments with a natural soil containing highly chlorinated hydrocarbons, Electrochim. Acta 47 (2002) 1405-1414.

[22] R. Lopez-Vizcaino, C. Saez, E. Mena, J. Villasenor, P. Canizares, M.A. Rodrigo, Electro-osmotic fluxes in multi-well electro-remediation processes, J. Environ. Sci. Health, Part A: Toxic/Hazard. Subst. Environ. Eng. 46 (2011) 1549-1557.

[23] S.S. Kim, S.J. Han, Application of an enhanced electrokinetic ion injection system to bioremediation. Water, Air, Soil Pollut. 146 (2003) 365-377.

[24] W. Xu, C. Wang, H. Liu, Z. Zhang, H. Sun, A laboratory feasibility study on a new electrokinetic nutrient injection pattern and bioremediation of phenanthrene in a clayey soil, J. Hazard. Mater. 184 (2010) 798-804.

[25] M. Pazos, E. Rosales, T. Alcántara, J. Gómez, M.A. Sanromán, Decontamination of soils containing PAHs by electroremediation: A review, J. Hazard. Mater. 177 (2010) 111.

[26] H.I. Gomes, C. Dias-Ferreira, A.B. Ribeiro, Electrokinetic remediation of organochlorines in soil: Enhancement techniques and integration with other remediation technologies, Chemosphere 87 (2012) 1077-1090. 
[27] R.T. Gill, M.J. Harbottle, J.W.N. Smith, S.F. Thornton, Electrokinetic-enhanced bioremediation of organic contaminants: A review of processes and environmental applications, Chemosphere 107 (2014) 31-42.

[28] L. Cang, Q.-Y. Wang, D.-M. Zhou, H. Xu, Effects of electrokinetic-assisted phytoremediation of a multiple-metal contaminated soil on soil metal bioavailability and uptake by Indian mustard, Sep. Pur. Technol. 79 (2011) 246-253.

[29] L. Cang, D.-M. Zhou, Q.-Y. Wang, G.-P. Fang, Impact of electrokinetic-assisted phytoremediation of heavy metal contaminated soil on its physicochemical properties, enzymatic and microbial activities, Electrochim. Acta 86 (2012) 41-48.

[30] K.R. Reddy, K. Maturi, C. Cameselle, Sequential Electrokinetic Remediation of Mixed Contaminants in Low Permeability Soils, J. Environ. Eng. 135 (2009) 989-998.

[31] E. Mena, P. Rubio, P. Cañizares, J. Villaseñor, M.A. Rodrigo, Electrokinetic transport of diesel-degrading microorganisms through soils of different textures using electric fields, J. Environ. Sci. Health, Part A: Toxic/Hazard. Subst. Environ. Eng. 47 (2012) 274-279. 
Table 1. Properties of the kaolinite

\begin{tabular}{lc}
\hline \multicolumn{2}{c}{ Mineralogy (dry weight) } \\
\hline $\mathrm{Al}_{2} \mathrm{O}_{3}$ & $34.5 \%$ \\
$\mathrm{CaO}$ & $0.1 \%$ \\
$\mathrm{Fe}_{2} \mathrm{O}_{3}$ & $0.6 \%$ \\
$\mathrm{~K}_{2} \mathrm{O}$ & $0.7 \%$ \\
$\mathrm{SiO}_{2}$ & $52.4 \%$ \\
$\mathrm{TiO}_{2}$ & $0.3 \%$ \\
$\mathrm{Others}^{2}$ & $11.4 \%$ \\
\hline \multicolumn{2}{c}{ Particle size distribution } \\
\hline Clay & $78.0 \%$ \\
Sand & $4.0 \%$ \\
Silt & $18.0 \%$ \\
\hline \multicolumn{2}{c}{ Other properties } \\
\hline Hydraulic conductivity & $10^{-7} \mathrm{~cm} \cdot \mathrm{s}^{-1}$ \\
Organic content & $0.0 \%$ \\
Specific density & 2.6 \\
pH & 4.9 \\
\hline
\end{tabular}

